Nils Elander • Mikhail Volkov • Åsa Larson •

Michael Stenrup · J. Zsolt Mezei • Evgeny Yarevsky •

Sergey Yakovlev

\title{
Quantum Scattering with the Driven Schrödinger Approach and Complex Scaling
}

Received: 4 December 2008 / Accepted: 13 January 2009 / Published online: 21 March 2009

(C) Springer-Verlag 2009

\begin{abstract}
Quantum scattering calculations of two and three-body systems with Coulomb interaction using the driven Schrödinger equation combined with exterior complex scaling are discussed. A rigorous formulation for two-body scattering is reported, and its generalization to three-body scattering is considered.
\end{abstract}

\section{Introduction}

The understanding of the dynamics of small molecular systems have made a considerable progress during the past 10 years. The identification of the measured peak in the cross section of the reaction $\mathrm{F}+\mathrm{HD}\left(v^{\prime}, j^{\prime}\right) \rightarrow \mathrm{FHD} \rightarrow \mathrm{FH}\left(v^{\prime \prime}, j^{\prime \prime}\right)+\mathrm{D}$ as an isolated resonance would not have been possible if not for the simultaneous theoretical and computational developments described in [1]. A number of other experimental and theoretical studies demonstrate the existence of resonances in several primary chemical reactions [2,3]. Theoretical methods to describe these reactions were created by Manolopoulos et al. and are reviewed in [2,3]. Most of these methods are based on the hyperspherical approach and are limited to at most a few potential energy surfaces and neutral reactants and products.

Naturally abundant reactions may also include charged reactants or products. Some such processes may be studied in the double electrostatic storage ring DESIREE [4], which is currently being built at Stockholm university. The mutual neutralization reaction $\mathrm{H}^{+}+\mathrm{H}^{-} \rightarrow \mathrm{H}_{2}^{*} \rightarrow \mathrm{H}(1)+\mathrm{H}(n)$ [5], where $n$ is a principal quantum number, will be one of the first experiments in this ring. The charge transfer reaction $\mathrm{N}^{3+}+\mathrm{H} \rightarrow \mathrm{NH}^{3+} \rightarrow \mathrm{N}^{2+}+\mathrm{H}^{+}[6]$ is another candidate for early experiments. Another interesting experiment is the similar three-body reaction $\mathrm{H}_{2}^{+}+\mathrm{H}^{-} \rightarrow \mathrm{H}_{3}^{*} \rightarrow \mathrm{H}_{2}+\mathrm{H}$. A theoretical-computational study of this reaction can be performed using a method that can describe many-channel, three-body scattering of charged particles in a full angular momentum framework (MCTSCPFAM) which includes the features of Refs. [5,6].

This article is based on the presentations by M. Volkov and N. Elander at the Fifth Workshop on the Dynamics and Structure of Critically Stable Quantum Few-Body Systems, Erice, Italy, 2008.

N. Elander $(\varangle) \cdot$ M. Volkov $\cdot$ Å. Larson · M. Stenrup · J. Z. Mezei · E. Yarevsky · S. Yakovlev

Molecular Physics Division, AlbaNova University Center, Stockholm University, 10691 Stockholm, Sweden

E-mail: elander@physto.se

E-mail: miha@physto.se

E-mail: aasal@physto.se

E-mail: stenrup@physto.se

E-mail: mezeijzs@physto.se

E. Yarevsky $\cdot$ S. Yakovlev

Department of Physics, St. Petersburg State University, St. Petersburg, Russia

E-mail: yarevsky@gmail.com

E-mail: sl-yakovlev@yandex.ru 
In a well quoted short paper Nuttal and Cohen [7] propose the use of the driven Schrödinger equation combined with complex scaling [8] to compute quantum cross sections for short range potentials. Rescigno et al. [9] have recently explored this method [7] combined with exterior complex scaling [8] (ECS) in several electron-atom (molecule) scattering studies.

The present study is focused on developing theoretical and computational methods for MCTSCPFAM. The driven Schrödinger approach is discussed in Sect. 2. In the following Sect. 3 we give an outline of a new proof showing that the method of [9], in the two-body case, can be rigorously generalized to include a potential composed of a short range potential and a long range Coulomb contribution. For a more complete discussion of this work we refer to recent work of Volkov et al. [10]. We then outline how to generalize the method [10] to a many channel, three-body problem in Sect. 4.

\section{The Driven Schrödinger Equation: an Introduction}

The idea in [7] was to consider the Schrödinger equation and split the wave function into an incoming $\Psi_{\text {in }}$ and a scattered $\Psi_{\text {scat }}$ part, $\Psi=\Psi_{\text {in }}+\Psi_{\text {scat }}$. As the incoming wave satisfies the free Schrödinger equation, we find

$$
(H-E) \Psi=0 \quad \Rightarrow \quad(H-E) \Psi_{\text {scat }}=-(H-E) \Psi_{\text {in }}=-V \Psi_{\text {in }},
$$

where $V$ is the potential energy. With given boundary conditions, including ECS we can compute the scattered wave for an arbitrary energy $E$. Following [7] one can use complex scaling to obtain zero boundary condition at infinity if the potential there decreases exponentially or faster. Rescigno et al. [9] realized, without presenting rigorous arguments, that this method could be extended to long-range potentials. Scattering quantities like the amplitude and the $S$-matrix can then be computed. Below we use atomic units.

\section{The Two-Body Problem for Coulomb Plus Short-Range Potentials}

Let the reduced mass be one. The partial wave $\Psi_{\ell}(k, r)$ with angular momentum $\ell$ satisfies the radial Schrödinger equation $\left(H_{\ell}+V-k^{2}\right) \Psi_{\ell}=0$. Here, the "free" Hamiltonian is $H_{\ell}=-\partial_{r}^{2}+\ell(\ell+1) / r^{2}$ and the interaction potential $V(r)=2 q^{2} / r+V_{s}(r)$, where $q$ is a charge. The short-range potential $V_{s}$ is assumed to vanish faster than $1 / r^{2}$ at large $r$.

The asymptotic form of the scattering solution is defined in terms of the Coulomb wave functions [11]. Let $\eta=q^{2} / k$. The scattering amplitude $\mathcal{A}=e^{2 \mathrm{i} \sigma_{\ell}}\left(e^{2 \mathrm{i} \delta_{\ell}}-1\right) /(2 \mathrm{i})$, where $\sigma_{\ell}=\arg \Gamma(1+\ell+\mathrm{i} \eta)$, is determined by the phase shift $\delta_{\ell}$, which is due to the presence of the potential $V_{S}$.

In order to reformulate the problem in terms of a driven Schrödinger equation, the potential $V$ is split into a sum of an interior part, $V_{R}$, and an exterior part, $V^{R}$, such that $V=V_{R}+V^{R}$. Here $R$ is a parametrical radius chosen such that $V(r) \ll E$ for $r \geq R$. The finite-range interior potential $V_{R}$ is introduced in such a way that $V_{R}=V$, if $r \leq R$, and $V_{R}=0$ otherwise. The splitting of the wave function $\Psi_{\ell}=\Psi_{R}+\Psi^{R}$ leads to the inhomogeneous equation

$$
\left(H_{\ell}+V-k^{2}\right) \Psi_{R}=-V_{R} \Psi^{R}
$$

provided that $\Psi^{R}$ obeys the Schrödinger scattering problem for the exterior potential

$$
\left(H_{\ell}+V^{R}-k^{2}\right) \Psi^{R}=0,
$$

with the corresponding amplitude $\mathcal{A}^{R}$. Equation (3) defines $\Psi^{R}$. For $r \leq R$ the potential $V^{R}$ vanishes $\left(V^{R}(r)=\right.$ $0)$. Therefore, the function $\Psi^{R}(k, r)$ must be proportional to the Riccati-Bessel function $\hat{j}_{\ell}$ :

$$
\Psi^{R}(k, r)=a^{R} \hat{j}_{\ell}(k r) .
$$

The function $\Psi^{R}(k, r)$ for $r>R$ can be expressed in terms of Jost solutions [11]. The requirement for the wave function and its derivative to obey the continuity conditions at the point $r=R$ completes the construction of $\Psi^{R}$. This construction provides a way to calculate $a^{R}$ and the amplitude $\mathcal{A}^{R}$, defined through the relation $\mathcal{A}^{R}=e^{2 \mathrm{i} \sigma_{\ell}}\left(e^{2 \mathrm{i} \delta^{R}}-1\right) /(2 \mathrm{i})$. 
Once the wave function $\Psi^{R}$ has been constructed, (2) is well defined. By imposing the boundary conditions

$$
\Psi_{R}(k, 0)=0, \quad \Psi_{R}(k, r) \sim \mathcal{A}_{R} u_{\ell}^{+}(\eta, k r), \quad r \rightarrow \infty,
$$

where $u_{\ell}^{+}=e^{-\mathrm{i} \sigma_{\ell}}\left(G_{\ell}+\mathrm{i} F_{\ell}\right)$ is the sum of the regular and irregular Coulomb wave functions, this equation determines the remainder of the scattering wave function $\Psi_{R}=\Psi_{\ell}-\Psi^{R}$. As we solve the boundary value problem $(2,5)$ for $\Psi_{R}$ on the interval $[0, R]$ only, the function $\Psi^{R}$ is well-defined through (4). The amplitude $\mathcal{A}_{R}$ is given by $\mathcal{A}_{R}=\mathcal{A}-\mathcal{A}^{R}$. The representation of the amplitude $\mathcal{A}_{R}$ in terms of the residual phase shift $\delta_{R}=\delta_{\ell}-\delta^{R}$ has the standard form

$$
\mathcal{A}_{R}=e^{2 \mathrm{i}\left(\sigma_{\ell}+\delta^{R}\right)}\left(e^{2 \mathrm{i} \delta_{R}}-1\right) /(2 \mathrm{i})
$$

The boundary problem (5) becomes simpler when the radius $R$ is chosen large enough. If $k R \gg \ell(\ell+1)+\eta^{2}$, then the asymptotic form of $\Psi_{R}(k, r)$ reduces to

$$
\Psi_{R}(k, r) \sim \mathcal{A}_{R} e^{\mathrm{i}(k r-\ell \pi / 2-\eta \log 2 k r)} .
$$

For the phase shift $\delta^{R}$, we find the asymptotic behavior $\delta^{R} \sim \eta \log 2 k R-\sigma_{\ell}$. The asymptotic form (7) can be used in order to calculate the amplitude $\mathcal{A}_{R}$ in its local representation. Using the Green's function formalism, we can also find its integral representation

$$
\mathcal{A}_{R}=-k^{-1} \int_{0}^{R} d r^{\prime} \Psi^{R}\left(k, r^{\prime}\right) V\left(r^{\prime}\right)\left[\Psi^{R}\left(k, r^{\prime}\right)+\Psi_{R}\left(k, r^{\prime}\right)\right] .
$$

The success in solving the driven Schrödinger equation by ECS depends on whether the driving term vanishes for complex values of the radial coordinates. The driven Schrödinger equation formulation $(2,5)$ perfectly meets this requirement since the potential on the right hand side is of finite range. Another useful observation made from the representation (8) is that the scattering amplitude $\mathcal{A}_{R}$ is completely determined by that part of the solution $\Psi_{R}$, which is restricted on the finite domain $0 \leq r \leq R$. After the ECS transformation of the coordinate, the boundary condition (5) becomes the zero boundary condition, and the Schrödinger equation (2) can be easily solved.

Summarizing the description of our approach, we can say that the only parameter affecting the results is the radius $R$. It is important that the scattering problem (1) is exactly reduced to the boundary value problem on the interval $[0, R]$ for an arbitrary finite value $R[10]$. For the numerical calculations, however, we need to use the asymptotics of the wave function at the right boundary $R$. This gives an error, those magnitude is defined by the accuracy of the asymptotics.

In order to find the solution, we first ECS-transform and solve Eq. (2) with the zero boundary conditions and compute the function $\Psi_{R}(k, r)$. Then we find the amplitude $\mathcal{A}_{R}$ using the local representation (7) or with the integral representation (8). Using (6) with $\delta^{R}=\eta \log 2 k R-\sigma_{\ell}$, we calculate the phase shift $\delta_{R}$ and finally reconstruct $\delta_{\ell}$ with the relation $\delta_{\ell}=\delta^{R}+\delta_{R}$. The numerical implementation of this approach has recently been shown to have both good accuracy and high efficiency [10].

\section{The Driven Schrödinger Approach to the Three-Body Problem}

Three possible arrangements for an atom-diatom can be distinguished and denoted $\alpha=a, b, c$. Let the massscaled Jacobi coordinates $\mathbf{R}_{\alpha}$ and $\mathbf{r}_{\alpha}$ be the dissociative and diatomic coordinates, respectively. The total wave function $\Psi\left(\mathbf{R}_{\alpha}, \mathbf{r}_{\alpha}\right)$ of the system is given as the solution to the Schrödinger equation in one of the three Jacobi coordinate systems

$$
\left[-\Delta_{\mathbf{R}_{\alpha}}-\Delta_{\mathbf{r}_{\alpha}}+\sum_{\beta} V_{\beta}\left(\mathbf{r}_{\beta}\right)-E\right] \Psi\left(\mathbf{R}_{\alpha}, \mathbf{r}_{\alpha}\right)=0, \quad \alpha=a, b, c .
$$

Here, $V_{\beta}\left(\mathbf{r}_{\beta}\right)$ is the atom-atom interaction. The total wave function can be represented as the sum of the incoming wave and an unknown function $\Phi\left(\mathbf{R}_{\alpha}, \mathbf{r}_{\alpha}\right)$. The incoming wave describes the initial state of the system when the third particle is far away from the diatom in the rovibrational state $j$

$$
\Psi\left(\mathbf{R}_{\alpha}, \mathbf{r}_{\alpha}\right)=\Phi\left(\mathbf{R}_{\alpha}, \mathbf{r}_{\alpha}\right)+\varphi_{\alpha}^{j}\left(\mathbf{r}_{\alpha}\right) e^{\mathrm{i}\left(\mathbf{q}_{\alpha}^{j}, \mathbf{R}_{\alpha}\right)} .
$$


Here, the third particle momentum $q_{\alpha}^{j}$ is defined according to $E=\left(q_{\alpha}^{j}\right)^{2}-\epsilon_{\alpha}^{j}$, where $\varphi_{\alpha}^{j}\left(\mathbf{r}_{\alpha}\right)$ and $\epsilon_{\alpha}^{j}$ are the wave function and the energy of the diatom rovibrational state, respectively. Substituting representation (10) into Schrödinger equation (9), we get its inhomogeneous (driven) form

$$
\left[-\Delta_{\mathbf{R}_{\alpha}}-\Delta_{\mathbf{r}_{\alpha}}+\sum_{\beta} V_{\beta}\left(\mathbf{r}_{\beta}\right)-E\right] \Phi\left(\mathbf{R}_{\alpha}, \mathbf{r}_{\alpha}\right)=-\varphi_{\alpha}^{j}\left(\mathbf{r}_{\alpha}\right) e^{\mathrm{i}\left(\mathbf{q}_{\alpha}^{j}, \mathbf{R}_{\alpha}\right)} \sum_{\beta \neq \alpha} V_{\beta}\left(\mathbf{r}_{\beta}\right) .
$$

In order to find the solution to Eq. (11) the asymptotic boundary condition should be taken into account. This condition for large hyperradius $\rho=\sqrt{R_{\alpha}^{2}+r_{\alpha}^{2}}$ reads [12]

$$
\Phi\left(\mathbf{R}_{\alpha}, \mathbf{r}_{\alpha}\right) \sim \sum_{\alpha=a, b, c} \sum_{j}\left[A_{\alpha}^{j}\left(\hat{\mathbf{R}}_{\alpha}\right) \varphi_{\alpha}^{j}\left(\mathbf{r}_{\alpha}\right) \frac{e^{\mathrm{i} q_{\alpha}^{j} R_{\alpha}}}{R_{\alpha}}\right]+A_{\alpha}^{0}\left(\hat{\mathbf{r}}_{\alpha}, \hat{\mathbf{R}}_{\alpha}, \phi_{\alpha}\right) \frac{e^{\mathrm{i} \sqrt{E} \rho}}{\rho^{5 / 2}} .
$$

The amplitudes $A_{\alpha}^{j}, \quad j>0$ describe the elastic and rearrangement processes while $A_{\alpha}^{0}$ is the breakup amplitude.

The asymptotic form (12) has different forms in different arrangements. Therefore, its implementation into a practical numerical method for the solution of the three-body Schrödinger Eq. (11) is problematic. Hence, the use of the idea of the previous section leads in the three body case to drastic simplifications. If we apply ECS to the coordinates $r_{\alpha}, R_{\alpha}$ with some exterior radius, the scattered wave $\Phi\left(\mathbf{R}_{\alpha}, \mathbf{r}_{\alpha}\right)$ (12) vanishes exponentially. For exponentially decreasing potentials, the r.h.s. of Eq. (11) also vanishes, while for long-range potentials we need to use a technique similar to that outlined in the previous section. We then arrive at the equation (11) with zero boundary conditions at infinity, which can be solved with already developed methods.

Finally, we should extract the scattering amplitudes from the wave function $\Phi\left(\mathbf{R}_{\alpha}, \mathbf{r}_{\alpha}\right)$. The simplest way is to define the elastic and rearrangement amplitudes from the asymptotic boundary conditions. To do so, we choose the exterior radius large enough so that asymptotics (12) is already satisfied with sufficient accuracy. For smaller distances $R_{\alpha}$, the first terms in (12) dominate over the breakup term. For a chosen Jacobi coordinate frame (i.e., rearrangement channel $\alpha$ ), we project the wave function $\Phi$ onto $\varphi_{\alpha}^{j}\left(\mathbf{r}_{\alpha}\right)$. As the overlap integral of $\varphi_{\alpha}^{k}\left(\mathbf{r}_{\alpha}\right)$ with $\varphi_{\beta}^{k}\left(\mathbf{r}_{\beta}\right)$ for $\alpha \neq \beta$ is zero, other arrangement channels do not contribute. In the same channel, the vibrational eigenfunctions are orthogonal implying

$$
\int d \mathbf{r}_{\alpha} \varphi_{\alpha}^{k}\left(\mathbf{r}_{\alpha}\right) \Phi\left(\mathbf{R}_{\alpha}, \mathbf{r}_{\alpha}\right) \sim A_{\alpha}^{k}\left(\hat{\mathbf{R}}_{\alpha}\right) \frac{e^{\mathrm{i} q_{\alpha}^{k} R_{\alpha}}}{R_{\alpha}} .
$$

This equation gives both the elastic and rearrangement amplitudes.

A numerical generalization of the present MCTSCPFAM theory could be accomplished with the framework of our three-body finite element method [13] (ECSFEMFAM). The three-body eigenvalue problem for the bound state wave function $\Phi\left(\mathbf{R}_{\alpha}, \mathbf{r}_{\alpha}\right)$ is there formulated in terms of Eq. (11) with r.h.s. put to zero. The coordinate space is described by a set of rectangular finite elements. The solution wave function to the eigenvalue is described as the expansion over the FEM basis function $f_{i}, \Phi=\sum_{i} c_{i} f_{i}$, i.e., as an eigenvector $\mathbf{c}$. In this code the Hamiltonian $\mathbf{H}$ is expressed as finite element matrix elements $H_{i j}$, and the finite element overlap matrix is expressed as $S_{i j}$. If the inhomogeneous term in the r.h.s. of Schrödinger equation (11) is represented as a vector $\mathbf{b}$, we can formulate the eigenvalue problem, EP, and the driven Schrödinger equation scattering problem, DP, as

$$
\mathrm{EP}: \quad \mathbf{H c}=E \mathbf{S c} \quad \mathrm{DP}:(\mathbf{H}-E \mathbf{S}) \mathbf{c}=\mathbf{b}
$$

Starting with the ECSFEMFAM code we need to add the $\mathbf{b}$ term to compute the scattering wave function in terms of $\mathbf{c}$. The amplitudes are then obtained from a numerical realization of Eq. (13).

\section{Summary}

We have here outlined the use of the driven Schrödinger equation combined with ECS in order to treat quantum scattering of few particles. A rigorous formulation for two-body scattering is reported, and its generalization to three-body scattering is considered. Finally, we indicate how the theory may be realized numerically based on an existing finite element parallel code which is suitable for several potential energy surfaces. 
Acknowledgments This work is supported by Swedish Research Council. EY and MV acknowledges support from the Swedish Institute, ZM acknowledges support from Wenner Gren Foundation and MS, EY and SY acknowledge support from Stockholm University.

\section{References}

1. Liu, K., Skodje, R.T., Manolopoulos, D.E.: Resonances in bimolecular reactions. Phys. Chem. Commun. 5, 27 (2002)

2. Lagana, A., Lendvay, G. (eds.): Theory of Chemical Reaction Dynamics. NATO Science Series, vol. 145. Kluwer, Dordrecht (2004)

3. Non-adiabatic Effects in Chemical Dynamics. Faraday Discussions, vol. 127 (2004)

4. Schmidt, H.T., et al.: Desiree as a new tool for insterstellar ion chemistry. Internat. J. Astrobiol. 7, 205 (2008)

5. Stenrup, M., Larson, A., Elander, N.: Mutual neutralization in low energy $\mathrm{H}^{+}+\mathrm{H}^{-}$collisions. Phys. Rev. A 79, 012713 (2009)

6. Shilyaeva, K., Yarevsky, E., Elander, N.: Identifying resonance structures in a scattering cross section using the $\mathrm{N}^{3+}+\mathrm{H}^{\rightarrow}$ $\mathrm{NH}^{3+} \rightarrow \mathrm{N}^{2+}+\mathrm{H}^{+}$reaction as an example. J. Phys. B At. Mol. Opt. Phys. 42, 044011 (2009)

7. Nuttal, J., Cohen, H.L.: Method of complex coordinates for three-body calculations above the breakup threshold. Phys. Rev. 188, 1542 (1969)

8. Hislop, P.D., Sigal, I.M.: Introduction to Spectral Theory. Springer, New York (1996)

9. Rescigno, T.N., Baertschy, M., Byrum, D., McCurdy, C.W.: Making complex scaling work for long-range potentials. Phys. Rev. A 55, 4253 (1997)

10. Volkov, M.V., Elander, N., Yarevsky, E., Yakovlev, S.L.: Solving the Coulomb scattering problem using the complex scaling method. EPL 85, 30001 (2009)

11. Newton, R.G.: Scattering Theory of Waves and Particles. Springer, New York (1982)

12. Faddeev, L.D., Merkuriev, S.P.: Quantum Scattering Theory of Several Particle Systems. Kluwer, Dordrect (1993)

13. Alferova, T., Andersson, S., Elander, N., Levin, S., Yarevsky, E.: Resonances in three-body systems studied by a full angular momentum, smooth exterior complex scaling finite element method. Few Body Syst. 31, 177 (2002) 